\title{
Designing a Playful Communication Support Tool for Persons with Aphasia
}

\author{
Abdullah Al Mahmud, Idowu I.B.I. Ayoola, and Jean-Bernard Martens \\ Department of Industrial Design, Eindhoven University of Technology \\ P.O. Box 513, 5600 MB Eindhoven, The Netherlands \\ \{a.al-mahmud, j.b.o.s.martens\} atue.nl
}

\begin{abstract}
Many studies have investigated ways to leverage communication with people with aphasia. Here, a new concept is developed for people with non-severe aphasia in a way that accesses the emotional and unaware layer of a conversation and then communicates certain information to the partner hence; introducing new dynamics and structure to a conversation. We present the concept with detailed design and expert evaluation results.
\end{abstract}

Keywords: Aphasia, Accessibility, Storytelling, Contextual interview, Assistive technology.

\section{Introduction}

Aphasia is an acquired communication disorder that impairs a person's ability to process language or to understand others often caused by brain injury or trauma. Aphasia affects language comprehension and generation, such that people's ability to express themselves verbally suffers [3]. People with Broca's aphasia or expressive aphasia usually can understand or read what other people say or write but they have problems in expressing themselves verbally and in writing. The consequence of aphasia is that people have problems maintaining contacts with their friends cannot participate in social exchange and eventually become passive and socially isolated.

There has been a growing interest in improving the quality of life for people with aphasia through technology intervention $[1,2]$. It has been observed that the use of assistive technology can reduce social isolation and improve independence. Therefore, new tools are emerging for specific purposes such as helping aphasics while cooking [6], using internet [1] etc. These applications support higher-level communication needs of aphasics. However, people have several other communication needs such as social closeness and information transfer [5]. Up to now augmentative and alternative communication (AAC) devices have focused mainly on helping aphasics with basic communication needs [4]. Though these AACs are mostly used during therapy period they are however not conversation tools. Therefore, we see an opportunity in assisting aphasics to participate in conversations by our proposed design.

In this paper we focus on the development of a supportive device to help leverage communication with a target group of aphasic people besides using online strategies. We explore possibilities for a new communication support tool and present a final 
design that creates a new layer of interaction within a conversation and hopes to reconstruct the flow of information and enhance the overall experience derived from a dialogue in an unobtrusive way. This was achieved with the use of a monitored stress ball to stimulate an unaware interaction and then providing a constructive feedback to the partner. An iteration approach is observed to investigate the topic and to further develop into a final design. In understanding the problem domain, the researcher began with literature research to know ways of aiding communication or social activities for people with Aphasia. Furthermore the psychological needs for the target groups were briefly identified to settle on an approach for creating a new product for them. Lastly, an experiment was conducted to observe the kind of behaviors that may emerge in a group with limited communication abilities similar to aphasia. Certain design interventions were derived from these investigations and a final design opportunity was selected for development.

\section{Understanding the Problem Domain}

At the start of the project we investigated several questions such as a) How can we aid communication or social activities for aphasic patients? b) Can a group of people with impaired communication problems perform a common goal and how? An experiment designed for 15 participants was conducted to answer the questions. The objective was to make people work together using a square game by allowing them to perform physical tasks that oblige them to communicate and cooperate. All participants were students in their early twenties and had no impaired ability however the were not allowed to talk during the experiment depending on the type they belonged.

Each participant was allocated as member of type "A" or "B". Members of type "A" were not allowed to talk throughout the activities while those in type "B" were allowed to talk. The experiment was divided into three groups with sorted members. Group 1 had five members belonging to type "B". Group 2 had three members of type "A" and two of type "B". Group three had four members as type "A". The table below summarizes the division.

Table 1. Group division and number of members

\begin{tabular}{l|ll}
\hline Group ID & Members of type "A" & Members of type "B" \\
\hline Group 1 & - & 5 \\
Group 2 & 3 & 2 \\
Group 3 & 4 (omitted one person) & - \\
\hline
\end{tabular}

After experimenting, a questionnaire was handed out to members of groups to obtain both quantitative and qualitative data. The questions emphasized on their experience in the group as different types and to know how they improvised in order to communicate with other members. Also, by observing a video of the experiment, more information was obtained. Results from the experiment shows that even if members of a group is not allowed to talk, the group can still perform certain activity together given a common goal. Members are able to work together using 
gestures, pointing, etc, to communicate with one another and still retain a level of involvement/satisfaction. Eye contact was hardly sustained especially with people that were not allowed to talk. As a result, communication between members was shallow but rather focused their attention on the given task. Furthermore, participants who were not allowed to talk appeared less dominant hence, the social cohesion within the group may be improved by providing clues to help them properly take turns in a dialogue. Pointing gestures was the most effective method used to communicate especially with members who were not allowed to talk. In chapter 3, these insights are developed into actual concepts.

\section{Design Interventions and Development}

An idea generation session was conducted to obtain more ideas from certain directions. Below are three design opportunities that were identified and the third was selected to be further developed.

Recap. This idea attempts to bring an aphasic in contact with others to talk about their daily experiences. A sound recording device is used to record sound in a daily context and later replayed to stimulate discussion with friends or family. At the instance of recording the sound, pictures related to the subject matter or context can be captured and are automatically tagged to the sound sample to support future discussion when sharing. Figure 1(top) illustrates this idea; two individuals used the device to record their daily activity and later met with others for social discussions.

Information Schema. In this concept a person with aphasia may re-create or organize photos / illustrations on a timeline to generate or represent stories which may support future communication with others as illustrated on figure 1(middle). By pointing at the pictures on the board, he may be able to tell his stories easily without having to recall the words. The picture shows an aphasic creating his story on his playground, and later used them to tell his story to a friend.

The mediator concept. The design opportunity here is to enhance the flow or contributions of members in a conversation that may be between a person with aphasia and his partner. The partner in this case can be a therapist, family or friend. Having identified "turn taking" as an important aspect of communication, the concept attempts to provide feedback to partners in order to establish a convenient moment to interrupt or give clues. This is conceptualized with the use of objects that affords tinkering like in a stress ball in order to cause subconscious interaction with the object. According to the mental activity or state of a person, their interaction with the object changes and this information can be abstracted and used to provide feedback to the partner. Hopefully, this information flow would introduce a new dynamics/structure into their conversation. Figure 1(bottom) illustrates two people talking and holding different objects each. Through the objects, partners can receive impulse from the other and may as well initiate certain impulse to the other. 


\section{Final Concept}

The design opportunity further developed is the mediator concept because it provides a rare opportunity in design for aphasics. It deviates from the traditional photo and sound often capitalized or abused within this context however, it is aimed to focus on a rich and interactive experience that may be derived from a dialogue. The following subtopics present the steps of developments.
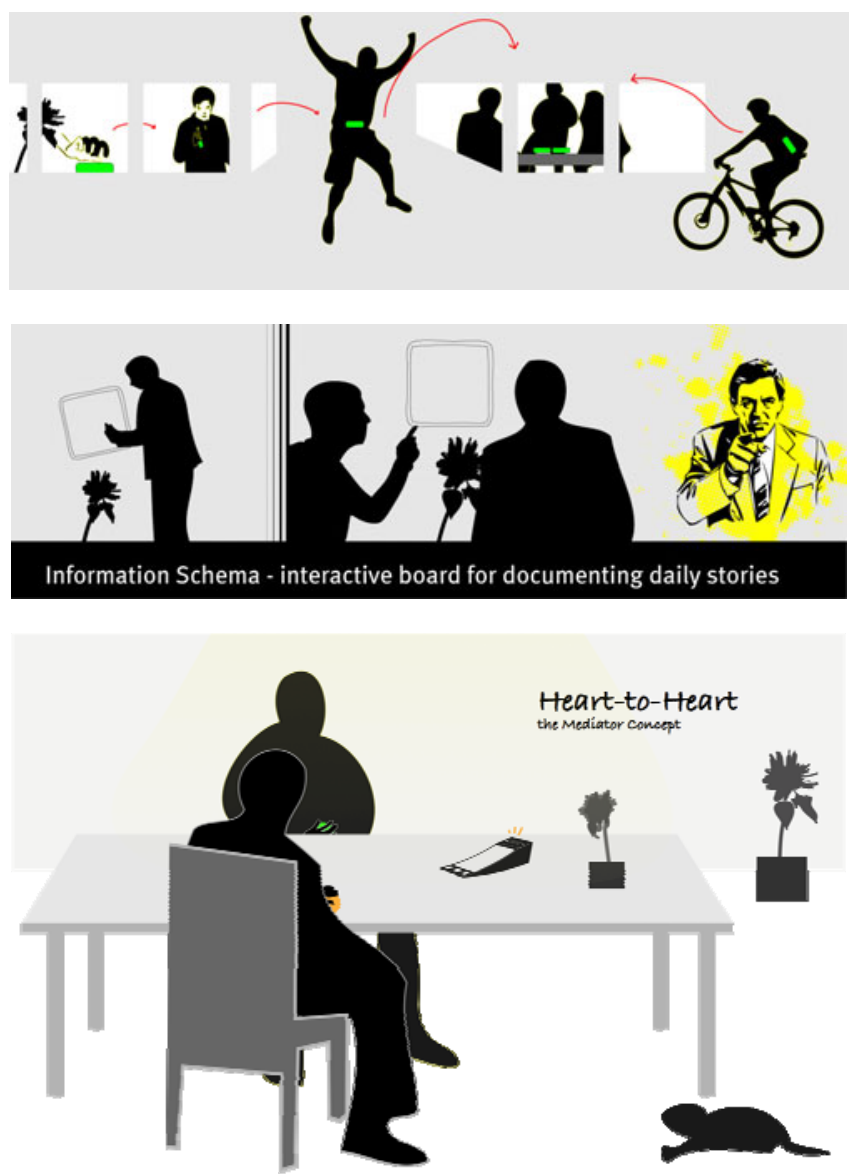

Fig. 1. Scenario of Audio Recap concept (top), scenario of Information Schema Recap concept (middle), scenario of Information Schema Recap concept (bottom)

A user test and concept evaluation was made to know how people unconsciously interact with objects in their hands and how much this interaction changes depending on the state on thinking. The prototype used for this investigation consists of two balloons inter-connected using a plastic tube. When one end is pressed, the pressure at the other end increases causing an instant feedback to the person holding at the other end. The first test was with two people, where one asked questions to the other. 
Different questions were asked and the difficulty level of the questions varied. It was observed that people unconsciously play with the balloon when they are relaxed. However, when the question demands more concentration, they momentarily stop playing with the ball.

\subsection{Material Explorations}

Throughout the design process, various materials were explored for building the ball. This is important to select the best material that would trigger the kind of interaction we need and to provide optimum comfort for the user. Examples of the materials created are silicon ball, balloon filled with powder, balloon filled with tinny wooden balls, tightly dense stress ball, PU ball, etc. PU ball was preferred over other materials because it allows full compression yet does not strain the hands. It is a perforated material and allows air to escape when pressed and then return to its original shape immediately after release. However due to unavailability of this material, silicon material was used. The silicon material provided similar affordances however do not allow full compression, as it is airtight.

\subsection{Digitizing Interaction and Feedback}

A silicon ball was made and monitored through the change in air pressure when pressed. A vibrator motor was used to provide instant feedback according to the results of the analysis. The prototype provides feedback in a way that makes the device seem invisible to users. Different feedback modalities were investigated however, haptic feedback was quickly settled upon in order to avoid visual distractions and to retain eye contact during the conversation. From empirical analysis, feedback in the rhythm of heartbeat was preferred over continuous vibration as it may create empathy amongst the partners. In essence, the harder the ball is pressed, the stronger the heartbeat, and when the user freezes while squashing the ball, the ball beats faster. A pilot test was done with some design students to ensure the prototype was robust enough for future testing and evaluations.

\subsection{Introducing a Desktop Interface}

A desktop interface (Fig. 2 top-left) is introduced and prototyped as part of the concept in order to provide visual cues to support the conversation. An example of the programs that may be installed may be a photo album organized in events. This way when a conversation began, a photo album can be opened to provide picture support. Another example may be a Phonetic \& Alphabet Chat to be used to scramble letters or sounds that may serve as clues. The purpose for linking the handheld device and the desktop devise is to strengthen the use of computers as visual or auditory cues. The handheld device provides information abstracted from the conversation in order to invite users to use the desktop device when needed. This may cause the desktop device and similar other computer applications built for support to have better utilitarian purpose. 

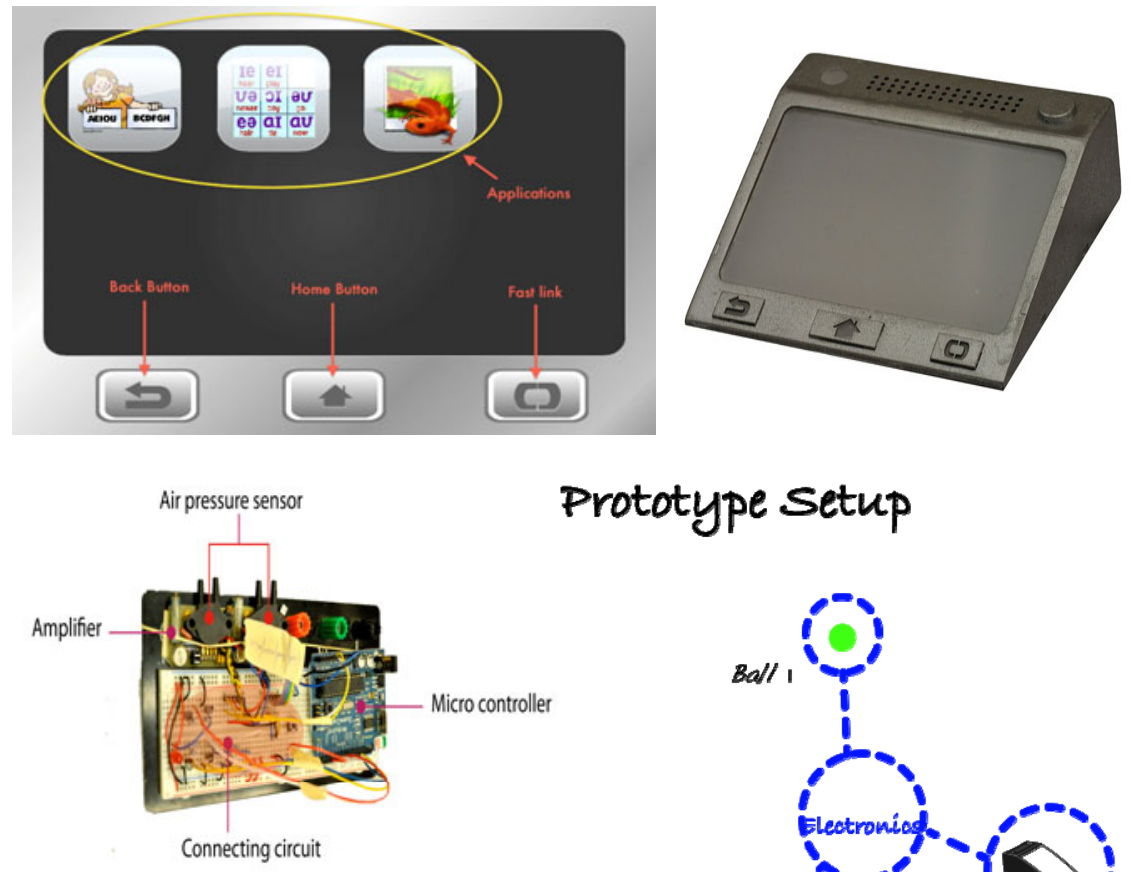

\section{Prototype setup}

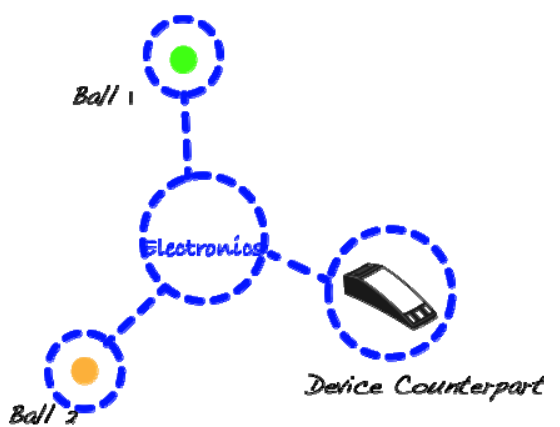

Fig. 2. The Desktop interface (top-left), the form of the desktop tool (top-right), the electronic prototype (bottom-left, and the prototype setup with balls (bottom-right)

\section{Expert Evaluation}

Having created a preliminary prototype to evaluate the design unity and rationale, a discussion session with an interaction expert was organized. The preliminary prototype was used to experience the design. The evaluation took place in an open office area. The working prototype was placed on a desk together with the desktop device. A computer was used to show the interface of the desktop device. Two chairs were placed closely apart to motivate a close proximity conversation. The goal was to enable the expert to experience the prototype in order to evaluate the rationale of the concept. This is aimed to inspire new insight for the design development.

Results: Interaction with objects of the design is classified into aware and unaware layers. The expert identifies interaction with the ball devices as unaware layer of communication as it is non-obtrusive to the users but yet would influence them. He believes it would change the quality of their conversation. However, when attention moves to the desktop device, the interaction with the system changes phase and causes the devices to become visible. He recommends developing the interaction and 
feedback with the ball devices because it may change people's expressive attitude and satisfaction in a dialogue.

i. The feedback received in form of heartbeat may have positive influence on users because it initiates the feeling of contact without physical touch. This may cause the partners to become more connected both physically and emotionally. He thinks there might be information overload if the person with aphasia regularly receives dynamic response from the device. This reveals the potential need to provide the users with some control; i.e., to choose to receive dynamic feedback from the device. His advice is to investigate the potentials of this design firstly within a therapeutic or structured conversation before moving on to general applications.

ii. The expert points out that users are constrained in movement especially because of the visible wires/connections. This may have psychological effect on users and the designed effect may be limited.

\section{Redesigning the Final Prototype: Mediator Concept}

The final prototype incorporates a wireless module and two ball devices for initiating and receiving impulse. It does not include the desktop device because the current line of development focus on the interaction and influence with the ball devices. Figure 3 is an overview of the final design. The wireless module includes a ball device and a case to hold the extended part of the electronics. It is implemented in a decentralized way, which makes it more sustainable, or standalone. On the casing is a button and a light source which shows the current state of the device. There are two selectable modes implemented on each module, one allows full functionality and the other allows the user to deactivate dynamic impulse meaning they would not receive regular impulse from the partner.

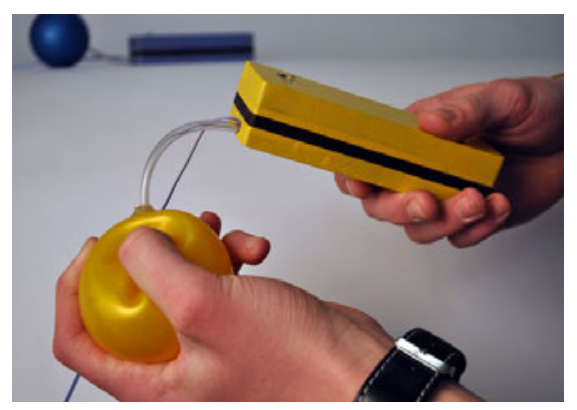

Fig. 3. The final prototype

Each module includes an air pressure sensor that measures the relative pressure in the ball to the atmospheric pressure. This signal is amplified within a range of 0-3.3 volts for analogue to digital conversion on an ATMega328 micro-controller chip. An interval routine that runs on the micro-controller processes this signal and outputs a 
modulation scheme/command through a wireless protocol to the second module. An interrupt routine is called when data arrives at the wireless port. Depending on the current mode of the device, the data is used to modulate the vibration motor embedded in the ball to stimulate haptic feedback.

\section{Conclusion and Future Steps}

Certain design interventions were derived and a course of development was settled upon which introduces a new dimension of a product for people with aphasia. The prospective product is a pair of wireless modules, which includes a ball in form of a stress ball, and a casing that holds parts of the electronics and controls. The end product was designed to access the unaware layer of a conversation in order to provide partners with a constructive feedback that may enrich the social experience of users.

Prototypes were created to validate certain design steps and later developed into a more experiential and advanced prototype, momentarily as a final design. Further investigations should be made to quantify the influence of the new product within a conversation. This would provide more insight for developments. In future, PU rubber material should be adopted, as it would enhance the interaction with the ball. Furthermore, investigations should be made to know if people are more likely to use a desktop application when the ball device is introduced. At this point, it is essential to evaluate the prototype with an aphasia therapist/expert to gain contextual insight; afterwards, a user test with actual users like aphasics may commence. An initial test between a person with aphasia and his/her therapist should be done before trying out in more dynamic situations. The design concept developed in this paper can eventually serve as an intervention tool, which can be integrated into application interfaces already built for people with aphasia.

\section{References}

[1] Egan, J., Worrall, L., et al.: Accessible internet training package helps people with aphasia cross the digital divide. Aphasiology 18(3), 265-280 (2004)

[2] Koppenol, T., Al Mahmud, A., Martens, J.-B.: When words fall short: helping people with aphasia to express. In: Miesenberger, K., Klaus, J., Zagler, W., Karshmer, A. (eds.) ICCHP 2010. LNCS, vol. 6180, pp. 45-48. Springer, Heidelberg (2010)

[3] Hillis, A.: Aphasia: progress in the last quarter of a century. Neurology 69(2), 200-213 (2007)

[4] Hux, K., Manasse, N., Weiss, A., Beukelman, D.: Augmentative and alternative communication. In: Chapey, R. (ed.) Language Intervention Strategies in Adult Apha-sia, pp. 675689. Williams \& Wilkins, Baltimore (2001)

[5] Light, J.: Interaction involving individuals using augmentative and alternative communication systems: State of the art and future directions. Augmentative and Alternative Communication 4(2), 66-82 (1988)

[6] Tee, K., Moffatt, K., et al.: A visual recipe book for persons with language im-pairments. In: Proc. CHI 2005, pp. 501-510. ACM, New York (2005) 